\title{
L'articulation technologies - organisations : des pistes pour une approche communicationnelle
}

Carole Groleau et Anne Mayère

\section{(2) OpenEdition}

1 Journals

Édition électronique

URL : http://journals.openedition.org/communicationorganisation/176

DOI : 10.4000/communicationorganisation. 176

ISSN : $1775-3546$

Éditeur

Presses universitaires de Bordeaux

Édition imprimée

Date de publication : 1 juillet 2007

Pagination : 140-163

ISSN : 1168-5549

\section{Référence électronique}

Carole Groleau et Anne Mayère, «L'articulation technologies - organisations : des pistes pour une approche communicationnelle », Communication et organisation [En ligne], 31 | 2007, mis en ligne le 01 juillet 2010, consulté le 19 avril 2019. URL : http://journals.openedition.org/

communicationorganisation/176 ; DOI : 10.4000/communicationorganisation.176 
Dossier : Migrations conceptuelles. D'où viennent les concepts de la communication organisationnelle?

\title{
Résumé
}

Tant dans la manifestation concrète du phénomène que dans la manière de les appréhender conceptuellement, les chercheurs interpellés par les technologies dans les organisations ont été confrontés à la fois à de nouvelles questions et à de nouveaux courants d'analyse au cours des deux dernières décennies. Dans un premier temps, nous envisageons les approches des TIC en organisation mobilisant la théorie de la structuration. Dans un deuxième temps, nous précisons en quoi la théorie de l'activité, qui présente des filiations avec les courants d'analyse précédents, a cherché à dépasser certaines de leurs limites. Nous envisagerons en conclusion les pistes ouvertes par des travaux récents relatifs aux technologies, pour considérer leur contribution à la compréhension de l'articulation technologie organisation et cerner ainsi plus avant les caractéristiques de la communication organisante dans les organisations contemporaines.

Mots clés : TIC; organisation ; approche communicationnelle ; théorie de la structuration ; théorie de l'activité

\begin{abstract}
Researchers interested in technologies have been confronted, over the last two decades, to difficulties in apprehending their research object, as well as to the arrival of new conceptual frameworks to study them in organizations. Among them, Giddens' structuration has inspired numerous researchers. We examine the studies issued from this tradition and see how activity theory provides means to overcome some of the limitations identified from structurationnal analyses of technology. We conclude by proposing research avenues to better apprehend the technology-organization relationship.
\end{abstract}

Key words :ICT, organization; organizational communication ; structuration theory; activity theory

Carole Groleau est Professeure agrégée au Département de communication de l'Université de Montréal. Ses recherches portent sur la place des technologies en milieu organisationnel. Plus particulièrement, elle s'est intéressée à la dimension organisante de la technologie et aux dynamiques interactionnelles soutenant le processus de changement technologique à partir d'approches comme l'action située, la cognition distribuée, la théorie de l'activité et la structuration.

Anne Mayère est Professeure à l'Université Toulouse 3, IUT A. Elle est chercheure au LERASS dont elle anime l'équipe Changement Organisationnel, Communication et Qualité. Son domaine de recherche concerne les évolutions des activités et dispositifs d'information et de communication associées à la maturation des formes contemporaines de production et d'échange, en termes notamment de rationalisation et de construction d'une interchangeabilité organisationnelle et informationnelle. 


\section{L'articulation technologies - organisations : des pistes pour une approche communicationnelle Carole Groleau, Anne Mayère}

carole.groleau@umontreal.ca anne.mayere@iut-tlse3.fr

Les technologies actuelles, plus ouvertes et paramétrables, posent des défis nouveaux aux chercheurs conceptualisant l'articulation entre technologie, communication et information dans des collectifs tels que ceux qui prennent forme dans les organisations. Le caractère organisant de la production d'information et de la communication dans les organisations tend à se renforcer dans le contexte contemporain de mutation des formes de production et d'échange, dans une évolution vers ce que certains ont appelé le 'capitalisme cognitif' (Bouillon, 2003 ; 2005). Les transformations associées au plan des dispositifs et activités d'information et de communication s'expriment en termes de rationalisation des productions d'information, d'extension des productions collectives d'information, et d'une construction de la disponibilité fondée sur les dispositifs de communication (Mayère, 2006 ; Pène, 2005).

Tant dans la manifestation concrète du phénomène que dans la manière de les appréhender conceptuellement, les chercheurs interpellés par les technologies dans les organisations ont été confrontés à la fois à de nouvelles questions et à de nouveaux courants $\mathrm{d}$ 'analyse au cours des deux dernières décennies.

Depuis le début des années 1980, de multiples approches ont tenté d'appréhender cet objet de recherche aux contours souvent flous (Jouët, 2000). Ainsi, la popularisation du paradigme interprétatif dans les études sur les organisations (Putnam et Pacanowsky, 1983), la naissance de mouvements comme le Computer Supported Cooperative Work - CSCW (Greif, 1988) ainsi que les 'workplace studies' (Heath, Knoblauch et Luff, 2000), et la diffusion d'approches s'appuyant sur le constructivisme social (Corcuff, 1995) ont diversifié les cadres d'analyse mobilisés pour examiner ce phénomène.

Nous proposons d'explorer certains des principaux courants théoriques qui, au cours des vingt dernières années, ont été mobilisés comme ancrage à ces travaux, en interrogeant leur origine disciplinaire, leur contexte d'émergence, et en questionnant la façon dont ces caractéristiques participent à mettre en forme leur approche des phénomènes communicationnels dans les organisations. 
Dossier : Migrations conceptuelles. D'où viennent les concepts de la communication organisationnelle?

Dans un premier temps, nous envisagerons les approches des TIC en organisation mobilisant la théorie de la structuration. Ce courant d'analyse s'avère plus «communicationnel» qu'il ne semble à première vue, et on peut comprendre le succès qu'il a rencontré dans les travaux de communication organisationnelle.

Dans un deuxième temps, nous verrons en quoi la théorie de l'activité, qui présente des filiations avec les courants d'analyse précédents, a cherché à dépasser certaines de leurs limites. Ce cadre d'analyse popularisé par Engeström (1987) place le changement et la transformation des pratiques organisationnelles au cœur de son analyse à partir du concept de contradiction. Souvent mobilisé autour de problématiques liées aux technologies, il constitue un cadre d'analyse potentiellement très heuristique au regard des questions que se posent actuellement les chercheurs en communication organisationnelle.

Les TIC constituent de plus en plus le support de formalisation de la 'culture' de l'organisation, de ses normes et valeurs, des méthodes, connaissances et savoir faire qui y sont mobilisées. Les activités de communication, qui à la fois participent à finaliser la mise en forme de ces technologies, et qui sont mises en œuvre dans la pratique de ces technologies pour la conduite des activités, participent du caractère organisant de la communication organisationnelle. Nous envisagerons en conclusion les pistes ouvertes par des travaux récents relatifs aux technologies, pour considérer leur contribution à la compréhension de l'articulation technologie - organisation et cerner ainsi plus avant les caractéristiques de la communication organisante dans les organisations contemporaines (Feenberg, 2004 ; Simondon, 2005).

\section{Différents courants d'analyse pour étudier l'articulation technologie-organisation}

Les cadres d'analyse mobilisés pour appréhender le rapport technologie-organisation se sont diversifiés au cours des deux dernières décennies pour offrir une variété de pistes intéressantes provenant de différentes disciplines connexes à la communication.

Un des événements marquants dans l'étude des technologies au cours des années 1980 fut la constitution du mouvement du Computer Supported Cooperative Work. Ce vocable inventé par Irene Greif et Paul Cashman dans un symposium en 1984 deviendra l'étiquette qui représentera des chercheurs d'une variété de disciplines se réunissant 
dans le cadre de conférences annuelles à partir de $1986^{1}$ pour réfléchir au support fourni par la technologie au travail collectif. Il n'y a pas de définition unanimement acceptée du mouvement CSCW. Dans les actes de la conférence du CSCW de 1988, Lucy Suchman, figure emblématique de ce mouvement le décrivait dans les termes suivants : « (le CSCW) a généré autant de questions qu'il a été capable d'en répondre dans le domaine du design de la technologie et du travail que cette technologie tente de supporter.»(p.V $)^{2}$. En marge de cet intérêt central pour le design des technologies, plusieurs chercheurs ont développé des axes de recherche sur la coopération en milieu de travail, la constitution d'espaces d'information partagée ainsi que sur les ajustements mutuels dans les milieux de travail informatisés.

Par la suite, des chercheurs intéressés par les pratiques de travail vont se regrouper pour former un nouveau mouvement nommé les workplace studies (Heath, Knoblauch et Luff, 2000 ; Luff, Hindmarsh et Heath 2000). Même si ces auteurs empruntent une variété de cadres théoriques, dont plusieurs ont déjà été associés au $\mathrm{CSCW}$, comme l'action située, la cognition distribuée et la théorie de l'activité, ils partagent un intérêt commun pour les dimensions technologiques et sociales du travail : «Ces études se penchent sur l'organisation sociale et interactionnelle d'activités en milieu de travail ainsi que sur la manière dont les outils et technologies, que ce soit des documents papier ou des systèmes multimédia complexes, s'imbriquent dans le travail au jour le jour et la collaboration $»^{3}$ (Heath, Knoblauch and Luff 2000, p 299).

Dans le champ même de la communication organisationnelle, de nouvelles approches allaient aussi ouvrir la voie à d'autres manières de cadrer le phénomène de l'informatisation. En 1983, Putnam et Pacanowski publiaient l'ouvrage pionnier des études interprétatives dans notre champ: Communication and Organizations: an Interpretive Approach. Dans cet ouvrage collectif, Weick soumet un agenda de recherche dans lequel, entre autres, il propose de lier les approches subjectivistes et objectivistes pour mieux comprendre la construction $\mathrm{du}$ processus soutenant les collectifs ; il suggère

\footnotetext{
${ }^{1}$ En réalité, les conférences $\mathrm{CSCW}$ et $\mathrm{ECSCW}$ alternent, ce qui permet d'accueillir les conférences en Europe, une année sur deux.

2 " $(\mathrm{CSCW})$ has opened as many questions as it has answered concerning the process of computer system design and the nature of the work that we mean to support".

3 "These studies address the social and interactional organization of workplace activities and the ways in which tools and technologies, ranging from paper documents through to complex multimedia systems, feature in day to day work and collaboration".
} 
Dossier : Migrations conceptuelles. D'où viennent les concepts de la communication organisationnelle?

également d'examiner différents niveaux d'analyse dans l'étude des organisations. C'est surtout cette dernière suggestion de Weick qui a inspiré Poole \& McPhee ; dans ce même ouvrage, ils empruntent au sociologue britannique Giddens la théorie de la structuration pour étudier le climat organisationnel ${ }^{4}$. Depuis, la théorie de la structuration a surtout été reprise pour examiner l'articulation technologie-organisation.

Ayant ainsi dressé une première cartographie de courants d'analyse importants qui ont structuré le champ de la recherche relative aux relations entre technologies et organisations, nous allons explorer plus avant deux cadres théoriques, émanant de ces propositions, qui empruntent de nouvelles pistes de recherche dans différentes disciplines pour étudier les technologies en milieu de travail. Nous examinerons d'abord comment la théorie de la structuration a été mobilisée pour étudier la technologie au sein des organisations. Ensuite, nous étudierons les possibilités qu'offre la théorie de l'activité, présente à la fois dans les workplace studies et le CSCW, pour examiner ce même phénomène.

Pour chacune de ces deux approches théoriques, nous allons évaluer leurs apports pour les chercheurs en communication en étudiant les conceptualisations de l'organisation qu'elles proposent. Nous préciserons comment elles rendent compte spécifiquement de la présence des technologies mais aussi de l'évolution dynamique de ces mêmes organisations à travers des études problématisant le changement technologique.

\section{La théorie de la structuration : sa visée et son application aux organisations en cours d'informatisation}

Comme le soulignait Poole \& McPhee (1983), la théorie de la structuration et son bagage conceptuel permettent de saisir la construction sociale à la base des organisations en tenant compte de sa dynamique historique ainsi qu'en créant des ponts entre les niveaux micro et macro de celle-ci. Des chercheurs comme Orlikowski et Barley qui ont été des précurseurs dans l'abondante littérature mobilisant la structuration pour étudier l'implantation de technologies en milieu de travail, ont vu, dans la théorie de la structuration, la

\footnotetext{
${ }^{4}$ En France, de nombreux chercheurs se sont aussi intéressés à l'application de la théorie de la structuration aux organisations tels que Eraly (1988), Bouchiki (1998) et Roux (2003). De plus, notons diverses publications dont un ouvrage collectif publié par Autissier \& Wacheux (2000) et un numéro spécial de la Revue de Gestion des Ressources Humaines édité par Rojot en 1998.
} 
possibilité de réconcilier les visions objectiviste et subjectiviste de l'organisation que la structuration rendait possible : "Plutôt que de perpétuer la division entre les subjectivistes et les objectivistes au sein des auteurs qui étudient la technologie, nous défendons une intégration de ses deux positions. Nous croyons que le phénomène de l'informatisation a besoin d'être cadré dans une théorie plus générale des structures sociales et de l'action. La théorie de la structuration de Giddens nous offre les outils nécessaires pour formuler une telle théorie intégratrice ». (Orlikowski \& Robey, 1991, p. 146). ${ }^{5}$

Plus spécifiquement, la théorie de la structuration développée par Giddens tente elle-même de conjuguer deux approches traditionnellement opposées en sciences sociales: soit la phénoménologie et le structuralisme (Giddens, 1984).

Giddens s'intéresse à la production et la reproduction du système social qu'il tente de lier conceptuellement avec les êtres humains quotidiennement engagés dans un flot de multiples activités. Pour ce faire, il prête attention aux patterns d'interaction qui se dégagent de la conduite de leurs activités journalières. Ce sont ces patterns d'interaction qui, maintes fois reproduits dans le temps et dans l'espace, caractérisent le système social dont se dégagent des propriétés structurelles. Ainsi, les propriétés structurelles sont à la fois le médium et le résultat de l'action humaine : elles forment le cadre qui oriente l'action, mais leur existence même repose sur la production et la reproduction des patterns qui les sous-tendent. Cette influence mutuelle entre actions et propriétés structurelles, que Giddens identifie comme la dualité du structurel, est centrale à la théorie de la structuration. La structuration peut alors être définie comme le processus par lequel les propriétés structurelles sont produites et reproduites dans le temps et dans l'espace à travers la dualité du structurel. Selon Giddens, le structurel constitue une réalité immatérielle, au plan des connaissances incorporées. Le structurel ne prend pas de forme matérielle, ni dans un texte, ni dans un objet. La seule manifestation concrète du structurel se retrouve dans les traces mnésiques du cerveau humain. Le structurel est une entité qui s'actualise dans le système social que Giddens définit comme un

\footnotetext{
5 "Rather than perpetuate the intellectual divide between the subjectivists and objectivists who study information technology, we argue for an integration of these positions. We believe that the phenomenon of information technology needs to be examined seriously as part of a more general theory of social structure and action. Giddens' theory of structuration provides the basis for such an integrative theory".
} 
Dossier : Migrations conceptuelles. D'où viennent les concepts de la communication organisationnelle?

ensemble de pratiques sociales telles qu'elles se déroulent dans le temps et dans l'espace. ${ }^{6}$

Comment la technologie est-elle appréhendée au sein de la littérature puisant son inspiration dans la théorie de la structuration ? Barley (1986) lui attribue le statut d'objet social dont le sens est défini dans son contexte d'utilisation. Il écrit: «les technologies sont plutôt considérées comme des occasions qui participent à la dynamique sociale, qui, à son tour, modifie ou maintient les contours de l'organisation» ${ }^{7}$ (1986, p 81).

D'un autre côté, les chercheurs gravitant autour de Poole et DeSanctis appréhendent la technologie comme une entité qui donne une forme matérielle au structurel. Nous croyons comme Banks \& Riley (1993), que Poole et DeSanctis réifient la structure en l'associant de cette manière à la technologie. Cette réification se note aussi dans le modèle de la dualité de la technologie proposé par Orlikowski (1992). Cependant, cette chercheure a évolué au fil de ses études, elle a exploré différentes pistes de recherche visant à conceptualiser la technologie dans ce cadre théorique. En 1996, elle s'inspire des approches situées ${ }^{8}$ pour s'éloigner de sa position antérieure qui fait de la technologie une matérialisation de la structure. Elle y définit la technologie dans les termes suivants: « la conceptualisation de la technologie que nous allons utiliser... propose d'examiner la technologie ni comme une entité matérielle, ni comme une construction sociale mais plutôt comme un ensemble de contraintes et d'habiletés mise en oeuvre dans l'action par l'appropriation de ses caractéristiques $»^{9}$ (Orlikowski, 1996, p. 69).

Ce virage, plus orienté vers la pratique, qui l'amène à penser les interactions avec la technologie en fonction des propriétés structurelles qu'elles rendent possibles, va se poursuivre dans ses travaux ultérieurs. En 2000, elle poursuit sa réflexion et raffine sa position en stipulant que la technologie ne donne pas une forme matérielle aux structures, mais qu'à travers les interactions avec cet

\footnotetext{
${ }^{6}$ Pour une description plus exhaustive de la théorie de la structuration, nous invitons le lecteur à lire Rojot (1998) ou Giddens $(1984 ; 1994)$.

7 "Technology are better viewed as occasion that trigger social dynamics which in turn, modify or maintain an organization's contours".

${ }^{8}$ action située et cognition distribuée

9 "The conceptualization of technology drawn on here... posits technology not as physical entity or social construction, but as a set of constraints and enablements realized in practice by the appropriation of technological features".
} 
artefact - désignées désormais comme enactment $t^{10}$ au lieu d'appropriation - émergent des propriétés structurelles. Elle ajoute : «la technologie-en-pratique est utilisée pour désigner la structure mise en acte de manière routinière lorsque nous utilisons de façon récurrente une machine, technique, instrument ou gadget dans nos activités situées au quotidien » (p 408, italique dans l'original $)^{11}$.

Cette position lui permet focaliser son analyse sur l'actualisation de la technologie en contexte. Au lieu de privilégier la technologie pour étudier le processus de structuration, elle choisit l'agent humain comme point d'ancrage de son analyse. Si cette piste semble mieux adaptée à la théorie de la structuration, elle se révèle problématique au niveau de son opérationnalisation dans les écrits produits à ce jour. Nous unissons notre voix à Rose, Jones et Truex (2005) pour noter les difficultés qu'Orlikowski rencontre à intégrer la technologie dans une approche qui privilégie le point de vue de l'agent humain. La technologie et le potentiel d'agir qu'elle rend disponible par ses contraintes matérielles, qu'ils soient actualisés ou pas, sont complètement évacués de ses études. Ainsi, les analyses structurationnistes inspirées de la 'technologie-en-pratique' n'intègrent même pas une description de la technologie dont on étudie la mise en acte.

L'analyse structurationniste des organisations ouvre des pistes intéressantes aux chercheurs en communication organisationnelle. Tel que défendu par Weick (1983) et Poole et McPhee (1983), elle permet de lier les niveaux d'analyse microscopiques et macroscopiques pour rendre compte de la réalité organisationnelle à travers les patterns d'interaction qui s'en dégage. Alors que l'application empirique de ce cadre soulève encore quelques problèmes (Groleau 2000; Groleau 2002; Giordano \& Groleau 2004), on voit, à travers les écrits inspirés de la structuration des débats d'ordre plus philosophiques. La place de la technologie dans le processus constitutif du social reste problématique. Après avoir associé la durabilité matérielle de la technologie au structurel, les derniers travaux d'Orlikowski en viennent pratiquement à évacuer les caractéristiques propres aux technologies étudiées pour focaliser exclusivement l'analyse sur la pratique des agents humains. De manière plus générale, cette difficulté

\footnotetext{
10 'Enactment' un terme provenant des travaux de Weick (1979) signifie mettre en acte.

11 "a technology-in-practice, to refer to the specific structure routinely enacted, as we use the specific machine, technique, appliance, device, or gagdget in recurrent ways in our everyday situated activities".
} 
Dossier : Migrations conceptuelles. D'où viennent les concepts de la communication organisationnelle?

propre à la structuration nous pousse à nous questionner sur la place de la matérialité dans nos organisations et plus particulièrement sur la dimension organisante des interactions que nous entretenons avec les entités matérielles ${ }^{12}$.

La théorie de la structuration problématise la constitution du système social, et de manière plus particulière la façon dont ce système évolue dans le temps. Ainsi, les chercheurs intéressés par les transformations associées à l'intégration de nouvelles technologies en organisation n'ont pas hésité à mobiliser ce cadre pour rendre compte de cette dynamique. Au sein de cette littérature nous avons répertorié deux types de travaux.

D'abord Orlikowski (1996) offre une alternative aux approches du changement planifié en proposant de tenir compte de la dimension émergente ${ }^{13}$ qui caractérise les changements au sein des entreprises. Pour ce faire, elle examine, à partir du moment de son implantation, la manière dont les membres de l'organisation mobilisent la technologie et ce faisant participent à la création de nouveaux patterns d'interaction. Walsham (2002) de son côté se distingue d'Orlikowski dans la mesure où il ne problématise pas la constitution du système social, mais utilise plutôt le concept de contradiction chez Giddens (1984) pour comparer la conception et l'usage de technologies dans des milieux culturels différents. Les contradictions au sein de son étude prennent la forme de conflits entre les concepteurs et les usagers qui appartiennent à des cultures nationales différentes, dans lesquelles des dimensions comme l'organisation du temps, les styles cognitifs et managériaux divergent. L'auteur nous explique comment les agents

\footnotetext{
${ }^{12}$ Nous avons choisi de focaliser notre analyse principalement sur les travaux issus de la théorie de la structuration. Certaines autres approches, comme la théorie de l'acteur réseau, ont proposé un cadre conceptuel visant à mieux prendre en compte les entités matérielles. Nous invitons les lecteurs intéressés par ces deux approches à consulter le Scandinavian Journal of Information Systems Vol.17, no.1 2005. On y retrouve un débat où des auteurs comme Orlikowski (2005), Walsham (2005) et Holmström (2005) échangent sur la place de la matérialité dans les deux approches. Engeström a voulu se distinguer des travaux de Latour en comparant la théorie de l'activité avec celle de l'acteur réseau (Engeström et Escalante 1996 ; Engeström 1996). Fondamentalement, la théorie de l'acteur réseau apparaît comme attribuant une place très limitative au structurel en général, et plus spécifiquement, à la dimension organisationnelle des processus étudiés.

${ }^{13}$ L'émergence se distingue ici de l'usage qu'en fait Orlikowski en 2000 pour caractériser les dynamiques interactionnelles lorsqu'elle explique ce qui se produit quand l'agent humain manipule la technologie. En 1996, elle fait plutôt référence au changement émergent qu'elle oppose au changement planifié comme conceptualisation du changement au sein des organisations.
} 
réflexifs appartenant à chacune des deux cultures en viennent à retenir des orientations différentes pour régler les conflits qui les opposent.

Walsham et Orlikowski ont cadré la transformation des pratiques de travail découlant de l'informatisation en intégrant, à leur façon, différentes facettes de la structuration. Ils se distinguent aussi dans leurs choix d'unités temporelles et spatiales pour cadrer leurs analyses. Malgré tout, les deux auteurs découpent leur objet empirique de manière à tenir compte du changement à partir de son actualisation sans analyser la dynamique sociale soutenant la planification stratégique qui a mené au changement. Nous croyons que cette étape préalable est importante parce qu'elle permet de spécifier l'évolution du système social étudié en intégrant la dynamique particulière au sein des membres de l'organisation qui a mené au choix d'implanter une nouvelle technologie. De plus, la théorie de la structuration, qui est une théorie de la reproduction sociale, s'avère lacunaire dans l'analyse du changement et de ses différentes facettes. Si les deux chercheurs précédemment cités identifient les conséquences inattendues comme point d'ancrage de la transformation, ils n'arrivent pas à expliquer conceptuellement le processus par lequel ces conséquences ont mené à des pratiques différenciées dans tel ou tel contexte particulier. Ainsi, il aurait été utile, particulièrement pour un chercheur en communication organisationnelle, de mieux comprendre le processus d'évolution de certains patterns d'interaction alors que d'autres restent inchangés.

Nous croyons que la théorie de l'activité offre une alternative intéressante à la structuration en proposant des conceptualisations de la technologie ainsi que du changement et de la dynamique sociale qui peuvent combler certaines des faiblesses identifiées à ce jour dans la mobilisation de la théorie de Giddens.

\section{La théorie de l'activité : une approche liant technologie, contradiction et communication}

La théorie de l'activité a beaucoup été sollicitée, à ce jour, par les chercheurs intéressés par les problématiques liées au design technologique. Depuis quelque temps, elle effectue une percée dans les études sur les organisations (Blackler, Crump and McDonald 2000; Artemava and Freeman 2001; Hasan \& Gould 2001; Jarzabkowski 2003; Prenkert 2006; Wilhelm 2006 ; Groleau 2006a ; Foot \& Groleau (sous presse). Nous croyons que le bagage conceptuel des auteurs de la troisième génération de chercheurs travaillant avec la théorie de l'activité, représentée par Engeström et ses collègues finlandais 
Dossier : Migrations conceptuelles. D'où viennent les concepts de la communication organisationnelle?

(Engeström 2001), peut nous aider à bien saisir l'articulation technologie-organisation.

La théorie de l'activité propose les concepts nécessaires à la fois pour décrire l'activité et pour rendre compte de l'évolution de ces activités, en conférant ainsi une dimension dynamique au cadre théorique. $\mathrm{Ce}$ sont ces deux facettes du cadre théorique, mises en œuvre dans les travaux d'Engeström, que nous allons exploiter pour conceptualiser le phénomène qui nous intéresse.

Cette approche appréhende l'activité à travers un ensemble de concepts tel qu'illustré dans la figure suivante (Engeström 1987) :

outils

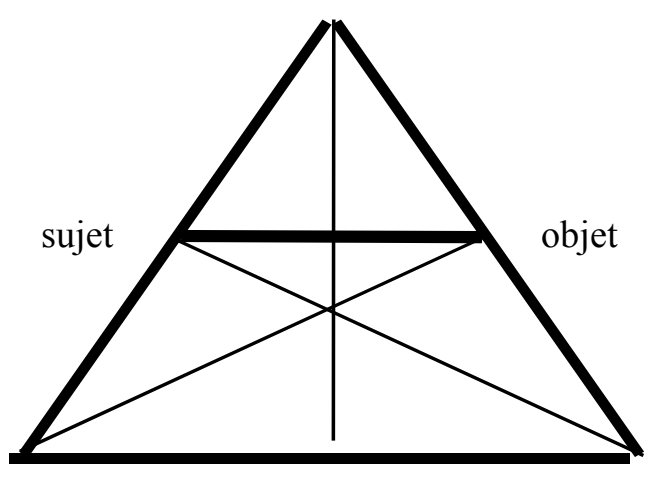

Règles

$$
\underset{\text { travail }}{\text { Communaú de Division du travail }}
$$

Figure 1. L'activité selon Engeström

La partie supérieure du schéma, liant sujets, outils et objet, est inspirée par les travaux de Vygotsky, fondateur de l'approche, et de ses collègues psychologues russes qui constituent la première génération de chercheurs associés à cette théorie

Dans ce schéma, les sujets désignent le ou les individus qui sont engagés dans la mise en œuvre de l'activité. De plus, le sujet représente le point de vue que le chercheur va privilégier dans son analyse. Le concept d'outil tiré des travaux de Vygotsky regroupe les outils techniques et psychologiques. Les outils techniques représentent les entités matérielles manipulées au quotidien, alors que les outils psychologiques font référence aux signes et aux codes traditionnellement associés au langage. Les deux catégories d'outils offrent les ressources qui permettent d'agir sur le monde en le 
transformant, et ainsi de lier les sujets à l'objet. L'objet désigne l'orientation de l'activité. Engeström (1999) distingue l'objet du but. Les buts sont conscients. Ils ont une durée de vie plutôt courte et orientent les actions individuelles. Les objets nécessitent le regroupement de plusieurs individus pour donner vie à l'activité. Au fur et à mesure de son actualisation, l'objet est appelé à être redéfini (Engeström 2001).

Cette dimension collective, qui caractérise l'objet et le distingue du but, est formalisée par Engeström (1987) dans la partie inférieure du triangle. Il s'est inspiré des travaux de Leont'ev qui a voulu élargir le cadre interindividuel du développement de l'activité du modèle de Vygotsky, pour s'intéresser au maillage des actions individuelles qui permettent à un collectif d'avoir un dessein commun, comme c'est le cas des organisations. Engeström a poursuivi la réflexion amorcée par Leont'ev et a intégré la dimension collective de l'activité dans une série de trois concepts : la communauté, les règles et la division du travail.

La communauté est composée de groupes et d'individus qui, sans participer directement à l'actualisation de l'activité, comme les sujets, influencent le déroulement de celle-ci. Pour illustrer cette distinction, Engeström utilise l'exemple d'une équipe de baseball. Les joueurs de l'équipe, qu'ils soient lanceur, receveur ou occupant tout autre position dans l'équipe, ont des actions individuelles qui contribuent à l'activité collective du jeu. Ce sont les sujets. Les membres de la communauté ne sont pas les joueurs directement engagés dans le jeu, mais les arbitres, les entraîneurs, les membres de l'auditoire qui contribuent à orienter le déroulement du jeu (Engeström 2000a). Comme le montre la figure plus haut, les règles, qu'elles soient explicites ou non, régissent les relations qui lient les sujets à la communauté. La division du travail est, comme les règles, un concept intermédiaire qui lie cette fois la communauté avec l'objet. La division du travail décrit comment les sujets engagés dans l'activité et la communauté modulent le 'faire'.

Parmi ces concepts qui spécifient les composantes de l'activité, on associe traditionnellement la technologie aux outils techniques. L'outil, dont la conceptualisation remonte aux travaux de Vygotsky, intègre et fait intervenir dans l'activité l'expérience historiquement construite de générations d'individus qui nous ont précédés. Par ce qu'il intègre, l'outil place l'activité dans un cadre spatio-temporel qui dépasse la situation immédiate. 
Dossier : Migrations conceptuelles. D'où viennent les concepts de la communication organisationnelle?

De plus on peut caractériser la technologie au titre d'outils techniques en fonction de la capacité d'agir qu'elle rend disponible aux sujets en fonction de l'objet qu'ils cherchent à atteindre ${ }^{14}$. Il serait toutefois réducteur de cantonner la technologie exclusivement aux outils dans le cadre de la théorie de l'activité. Dans la littérature associée à cette approche, on a repéré différentes propositions pour élargir la conceptualisation de la technologie au-delà de la catégorie des outils. Plus spécifiquement, Kuutti (1996) suggère d'explorer la conceptualisation de la technologie en fonction des règles, de la communauté et la division du travail, et de l'objet. Hasu \& Engeström (2001) ont considéré la technologie comme objet dans une étude récente. Ainsi, ils ont remarqué que pour les concepteurs d'une technologie qui tentent de comprendre la situation dans laquelle sera implantée la technologie qu'ils développent, la technologie n'est pas un outil mais bien l'objet du système d'activité des concepteurs. En tant que sujets, ils mobilisent des outils de toutes sortes et sont soutenus par une communauté qui guide leur agir collectif pour produire une nouvelle technologie.

Malgré ces ouvertures, les suggestions tendant à considérer la technologie comme une des autres composantes du triangle ont été peu suivies. Pourtant, les données que nous avons nous-mêmes colligées et analysées nous portent à croire que ces pistes sont fructueuses. Ainsi, dans une étude de cas effectuée auprès d'une firme d'architectes (Groleau 2006b), l'arrivée d'un nouveau logiciel de modélisation de perspectives en trois dimensions a nécessité que l'architecte senior, qui concevait ses perspectives avec crayon et papiers, se fasse aider d'un architecte-stagiaire pour les préparer désormais avec un ordinateur. Le stagiaire a rapidement étendu sa participation aux tâches de l'architecte senior. Alors que son activité devait se résumer à une aide technique, il a pris part lui aussi à la conception des nouveaux projets qui évoluent au fur et à mesure que la perspective se dessine. Ainsi cette tâche normalement réservée aux architectes seniors est maintenant partagée avec le stagiaire, créant ainsi une nouvelle division du travail au sein de l'équipe.

\footnotetext{
14 À ce titre, la technologie habilite et contraint simultanément le sujet qui le manipule. Les habiletés et contraintes associées à l'outil sont liées à l'agir. Ceci distingue la théorie de l'activité de la structuration au sein de laquelle Orlikowski (1996) a aussi défini la technologie en termes d'habiletés et de contraintes mais plutôt pour faire référence à la production d'interactions constituant le système social.
} 


\section{Conclusion : contributions et pistes d'approfondissement}

Si on compare la théorie de l'activité à la structuration, on réalise qu'elles partagent plusieurs points en commun. D'abord, les chercheurs qui ont mobilisé ces deux approches ont identifié le potentiel que présentaient ces cadres conceptuels pour surmonter la dualité opposant objectiviste à subjectiviste (Orlikowski \& Robey 1991 ; Barley 1986 ; Kuutti 1993). Toutes deux sont des approches constructivistes expliquant la constitution du collectif par une série d'interactions.

Ces deux cadres théoriques adoptant un regard constructiviste place la communication au cœur de leur étude du collectif. Ainsi c'est par la communication que les agents humains se constituent un système social avec la théorie de la structuration; de façon encore plus manifeste, dans la théorie de l'activité, c'est par la communication que le collectif passe à l'acte pour transformer son environnement tant matériel que social. Si les deux approches adoptent un regard communicationnel sur le collectif, elles privilégient différents axes de questionnement. La théorie de l'activité se distingue de la théorie de la structuration par cette volonté d'appréhender l'agir collectif, alors que le cadre de Giddens conceptualise plus l'être ensemble. Malgré cette différence dans la manière d'approcher le collectif, la théorie de l'activité tente de tenir compte de l'être ensemble soutenant l'activité à travers le concept de règles. Ce concept, à l'intersection du sujet et de la communauté, rend compte des normes et des rapports de domination qui régissent le collectif en action. Comme nous l'avons suggéré plus haut, il ne serait pas impossible d'imaginer que l'outil, outre son rôle de médiateur de connaissances construites dans le temps puisse aussi être appréhendé comme le médiateur de rapports de pouvoir et de manières d'être historiquement transmis.

La forte composante communicationnelle dans les processus soutenant le 'faire ensemble' conceptualisé dans la théorie de l'activité offre un agenda de recherche dans lequel l'action, les règles et la division du travail prennent forme par la communication entre humains autant qu'avec différents types d'outils. Ainsi on peut, à partir de la figure triangulaire proposée par Engeström (1987), questionner tant la constitution d'entités individuelles comme les outils, les règles, la division du travail, l'objet, que l'articulation de ces entités dans le faire ensemble. On peut par exemple interroger la façon dont les interactions sociales et matérielles se combinent dans l'atteinte d'un objet donné. 
Dossier : Migrations conceptuelles. D'où viennent les concepts de la communication organisationnelle?

Nous croyons que la théorie de l'activité offre des pistes particulièrement fructueuses pour examiner la place des technologies dans la conduite d'activité. Comme le défendait Vygotsky, les outils intègrent et permettent de faire circuler différentes formes de connaissances historiquement construites. De plus, comme nous l'avons abordé plus haut, les interactions avec les outils permettent de transformer l'environnement dans l'atteinte de l'objet. Ces deux dimensions de l'outil soulèvent des questions qui méritent d'être explorées empiriquement pour mieux saisir le potentiel de cet appareillage conceptuel qui demeure, selon nous, encore trop peu exploré.

De plus le concept d'interaction, au cœur de la théorie de l'activité, se distingue d'autres approches, comme celle de l'action située (Suchman 1987), qui la définit exclusivement en fonction de l'intelligibilité mutuelle qu'elle rend possible. La théorie de l'activité, comme la structuration, place l'interaction au service de la création de rapports de pouvoir et de normes. Ceci ouvre une série de questionnements liés à la constitution de règles, de normes et de division du travail par la communication.

Finalement, une des particularités de la théorie de l'activité est de spécifier le processus dynamique du système d'activité en l'appréhendant comme transformation impulsée par des contradictions récurrentes. L'identification des contradictions en tant que moteur des changements organisationnels conduit à mettre au centre du questionnement cette dynamique communicationnelle particulière à travers laquelle les points de vues se confrontent et sont appelés à être redéfinis collectivement.

Par sa logique interactionnelle, la théorie de l'activité permet de caractériser le changement organisationnel en mettant l'accent sur sa dimension communicationnelle. Ainsi, la théorie de l'activité nous aide à appréhender le changement comme un processus collectif par lequel les perspectives sur l'activité se confrontent dans l'interaction pour constituer un nouveau système d'activité. A ce jour, une seule étude sur la dimension communicationnelle de ce processus transformationnel a été effectuée par Engeström, Engeström et Kerosuo, 2003. Elle met en lumière les différents types de discours mobilisés dans des séances de travail au cours desquelles l'activité a été questionnée par les membres de l'organisation, suite à l'apparition de contradictions, et a été reconfigurée pour soulager la tension.

Les travaux de Giddens (1984) et d'Engeström (1987) sont fondés sur une épistémologie constructiviste, et interrogent les rapports sociaux. 
Ils partagent ces fondements avec les travaux de Feenberg, qui a pour sa part plus particulièrement exploré les définitions de la technologie. Or nous avons pu constater que de nombreux travaux en restent à une spécification très allusive de ce qui caractérise les technologies, et par là même échouent partiellement à problématiser les rapports entre technologies et organisations.

Feenberg propose une approche renouvelée de la technologie dépassant les approches essentialistes qui sont de loin dominantes. Ces approches tendent à considérer exclusivement « les techniques comme des dispositifs orientés vers l'efficacité » (Feenberg, 2004, p 14). En laissant close la 'boite noire' de ce qui constitue la technologie et ce qui intervient tant dans sa conception que dans son utilisation, le risque est grand d'alterner entre déterminisme technologique et déterminisme social, comme ceci a été indiqué s'agissant de divers travaux d'Orlikowski. En rester, comme dans de nombreux écrits, à la désignation de la technologie comme construit social ne fournit pas de cadre d'analyse opératoire.

C'est l'intérêt de l'apport de Feenberg qui propose de spécifier la technique comme processus de développement (et non comme état stable) constitué dans un double mouvement :

- celui de l'instrumentalisation primaire, qui participe à la réification de la pratique technique (ce que Simondon désignerait comme la constitution d'un tertium quid (2005)). C'est par elle que prend forme la dimension fonctionnelle des objets techniques ;

- celui de l'instrumentalisation secondaire, qui contribue à l'intégration de la technique dans son environnement et en assure le fonctionnement (le re-localise, dirait Giddens, après la délocalisation du premier mouvement).

Dans chacun de ces mouvements, Feenberg identifie quatre grands moments ou composantes. Sans pouvoir ici les spécifier, nous voudrions montrer en quoi chacun peut être considéré comme le nœud de rapports de force implicites ou explicites, de contradictions dirait Engeström, qui se jouent y compris dans l'exclusion de certains acteurs et groupes d'acteurs et par le monopole de l'intervention d'autres groupes. Ainsi dans le mouvement de l'instrumentalisation primaire, la décontextualisation consiste à séparer l'objet technique de son contexte d'émergence pour l'intégrer dans un système technique ; le réductionnisme consiste à simplifier cet objet, à le réduire aux aspects permettant de l'enrôler au mieux des fonctionnalités 
Dossier : Migrations conceptuelles. D'où viennent les concepts de la communication organisationnelle?

privilégiées. Dans le champ de l'organisation et des TIC, on peut ainsi voir en quoi l'identification de 'meilleures pratiques', qui sert notamment à modéliser les logiciels type ERP (Bazet, Mayère, 2004), passe par leur formalisation décontextualisée au regard de leurs lieux précis d'émergence; elle suppose également une sélection de l'information formalisée, au travers de l'identification des informations considérées comme 'primaires', et l'éviction d'informations dites 'secondaires'. Or la désignation de ce caractère primaire ou secondaire est relative aux acteurs, aux lieux, aux rapports de pouvoir, aux activités. Les directions générales et contrôleurs de gestion n'attendent pas les mêmes 'vues' et possibilités d'intervention que les personnels en charge des achats, de la production, ou des ressources humaines.

Le déploiement de nombre de technologies contemporaines fait émerger des conflits patents ou latents, en particulier s'agissant des TIC dans les organisations. On peut y voir la résultante d'un déni de légitimité, et la source d'une violence symbolique et pratique par le monopole que s'attribuent certains groupes d'acteurs dans la spécification des technologies.

Deux grandes dimensions ou moments de la communication organisationnelle sont concernées par ces enjeux.

La première a trait à la communication qui peut ou non avoir lieu, enrôlant différents groupes d'acteurs, lors de la spécification de l'objet technique dans ses fonctionnalités, ou lors du mouvement d'actualisation qui vise à en assurer l'intégration dans le contexte d'utilisation.

Selon le champ et les formes de la communication ayant eu lieu lors de la conception de l'objet technique - conception qui s'agissant des TIC est de plus en plus un processus récurrent - on peut faire l'hypothèse qu'en termes d'usage, les communications autorisées, favorisées ou ainsi rendues possibles par les TIC vont être différenciées. La seconde grande dimension est constituée des communications qui participent à mettre en forme l'information structurelle, les valeurs, le 'discours de la maisonnée' (d'Almeida, 2002), les méthodes, dont la formalisation participe au développement des technologies intellectuelles, ainsi que l'information et la communication mobilisées dans les pratiques. La logique de rationalisation qui prend appui sur les TIC participe à leur évolution.

A travers la mise en discussion de différents cadres d'analyse partageant des fondements épistémologiques structurants, il est ainsi 
possible de poser les bases d'une approche communicationnelle renouvelée de l'articulation entre technologies et organisation.

\section{Remerciements :}

La première auteure aimerait remercier le Conseil des Recherches Humaines et Sociales du Canada pour son support financier dans le cadre de cette recherche (CRSH410-2004-1091). De plus, nous aimerions exprimer nos remerciements aux évaluateurs pour leurs suggestions.

\section{Bibliographie}

d'Almeida N, Les promesses de la communication, PUF, 2002.

Autissier D, Wacheux F (dir.), Structuration et management des organisations: Gestion de l'action et du changement dans les entreprises. Paris, éd. L'Harmattan, 2000.

Artemava N, Freedman A, «Just the Boys Playing on Computers: An Activity Theory Analysis of Differences », Journal of Business and Technical Communication, 15(2), 2001, p. 164-194.

Bazet I, Mayère A, « Entre performance gestionnaire et performance industrielle, le déploiement d'un ERP », Sciences de la Société, ${ }^{\circ} 61$, 2004, p. 107-121.

Banks S P, Riley P, « Structuration Theory as an Ontology for Communication Research », Communication Yearbook, 16, 1993, p. 167-196.

Bardram J, «Designing for the Dynamics of Cooperative Work Activities », CSCW 98, 1998, p. 89-98.

Barley S R, «Technology as an Occasion of Structuring: Evidence from Observations of CT Scanners and the Social Order of Radiology Departments ", Administrative Science Quarterly, 31, 1986, p. 78-108.

Blackler F, Crump N, McDonald S, «Organizing Processes in Complex Activity Networks », Organization, 7(2), 2000, p. 277-300.

Bouchiki H, Structuration des organisations : Concepts constructivistes et étude de cas. Paris, éd. Economica, 1990.

Bouillon J.-L, « Les dimensions organisationnelles d'un changement de régulation: essai de mise en perspective communicationnelle », Actes du Forum de la Régulation 2003, Association Recherche et Régulation, Paris, 2003, actes sur cédérom 
Dossier : Migrations conceptuelles. D'où viennent les concepts de la communication organisationnelle?

Bouillon J.-L, «Autonomie professionnelle et rationalisations cognitives: les paradoxes dissimulés des organisations postdisciplinaires », Etudes de communication, n²8, 2005, p. 91-105.

Corcuff $\mathbf{P}$, Les nouvelles sociologies: constructions de la réalité sociale. Paris: Nathan, 1995.

DeSanctis G, Poole M S, "Capturing the complexity in advanced technology use: Adaptive structuration theory », Organization Science, 5(2), 1994, p. 122-147.

Engeström Y, Learning by Expanding: An Activity-Theoretical Approach to Developmental Research. Helsinki: Orienta-Konsultit Oy, 1987.

Engeström Y, «Interobjectivity, ideality and dialectics », Mind, Culture and Activity, 3(4), 1996, p. 259-265.

Engeström Y, "Communication, Discourse and Activity », Communication Review, 3(1-2), 1999, p. 165-185.

Engeström Y, «Activity Theory and the Social Construction of Knowledge », Organization, 7(2), 2000a, p. 301-310.

Engeström Y, «From Individual Action to Collective Activity and Back: Developmental Work Research as an Interventionist Methodology ", in Luff P, Hindmarsh J, Heath C (dir.), Workplace Studies: Recovering Work Practice and Information System Design, Cambridge, U.K.: Cambridge University Press, 2000b, p. 150-166.

Engeström Y, «Expansive Learning at Work: Toward an Activity Theoretical Reconceptualization », Journal of Education, 14(1), 2001, p. 133-156.

Engeström Y, Escalante V, « Mundane Tool or Object of Affection? The Rise and Fall of the Postal Buddy » In Nardi B (dir), Context and Consciousness: Activity Theory and Human-Computer Interaction Cambridge, MA: MIT Press, 1996, p. 325-373.

Engeström Y, Engeström R, Kerosuo H, « The Discursive Construction of Collaborative Care », Applied Linguistics, 24(3), 2003, p. 286-315.

Eraly A, La structuration de l'entreprise: La rationalité en action, Bruxelles: éditions de l'Université de Bruxelles, 1988.

Feenberg A, (Re)penser la technique, éd. La Découverte, MAUSS, 2004. 
Foot K A, Groleau C, (soumis) «Contradictions, Transitions and Organizational Development: A Dual-Motor Perspective Provided by Activity Theory », Organization Studies.

Giddens A, The Constitution of Society. Cambridge, MA: The Polity Press, 1984.

Giddens A, Les conséquences de la modernité, éd. L'Harmattan, 1994.

Giordano Y, Groleau C, «Institutionnalisation et théorie de la structuration: repenser les liens organisation-technologie », in Huault (dir.), Théories de l'institutionnalisation. Paris FNEGE, 2004.

Greif I (dir.), Computer-Supported Cooperative Work: A Book of Readings. San Mateo, CA: Morgan Kaufman, 1988.

Groleau $\mathbf{C}$, «La théorie de la structuration appliquée aux organisations: le cas des études sur les technologies ", in Autissier D, Wacheux F (dir.), Structuration et management des organisations: Gestion de l'action et du changement dans les entreprises, Paris: L'Harmattan, 2000, p. 155-179.

Groleau C, «Structuration, Situated Action and Distributed Cognition: Rethinking the Computerization of Organizations ", Système d'Information et Management, 2(7), 2002, p. 13-36.

Groleau C, «One Phenomenon, Two Lenses: Apprehending Collective Action From the Perspectives of Coorientation and Activity Theories ", in Cooren F, Taylor J R, Van Every E J (dir.), Communication as Organizing : Empirical and Theoretical Explorations in the Dynamic of Text and Conversation, Mahwah, NJ: Laurence Earlbaum, 2006a.

Groleau C, «Intégration de technologies de l'information et de la communication en milieu de travail: Penser l'arrimage des médiations sociales et matérielles avec la théorie de l'activité », Colloque Pratiques et usages organisationnels des sciences et technologies de l'information et de la communication, Rennes, 2006b, p. 24-30.

Hasan H, Gould E, "Support for the Sense-making Activity of Managers», Decision Support System, 31, 2001, p. 71-86.

Hasu M, Engeström Y, "Measurement in action: an activitytheoretical perspective on producer-user interaction », International Journal Human-Computer Studies, 53, 2000, p. 61-89. 
Dossier : Migrations conceptuelles. D'où viennent les concepts de la communication organisationnelle?

Heath C, Knoblauch H, Luff P, «Technology and Social Interaction: The Emergence of 'Workplace Studies », British Journal of Sociology, 51(2), 2000, p. 299-320.

Holmström J, «Theorizing in IS Research: What Came Before and What Comes Next? », Scandinavian Journal of Information Systems, 17(1), 2005, p. 167-174.

Jarzabkowski P, «Strategic Practices: An Activity Theory Perspective on Continuity and Change », Journal of Management Studies, 40(1), 2003, p. 23-55.

Kuutti K, "Work Activity as the Bridge Between Social and Technical », in Bowker G et alii (dir.), Proceedings of The Social Science Research Technical Systems and Cooperative Work Workshop. CNRS, Paris, 1993, p. 190-201.

Kuutti K, "Activity Theory as a Potential Framework for HumanComputer Interaction Research », in Nardi B A (dir.), Context and Consciousness: Activity Theory and Human-Computer Interaction, Cambridge, MA: MIT Press, 1996, p. 17-43.

Luff P, Hindmarsh J, Heath C, Workplace Studies: Recovering Work Practice and Informing System Design, New York, NY: Cambridge University Press, 2000.

Mayère A., "Les activités de communication et de production d'information dans les organisations. Apports et enjeux de la recherche en SIC », Actes du XVème Congrès des SIC, SFSIC, Bordeaux, 2006, p. 431 - 445.

Orlikowski W, « The Duality of Technology: Rethinking the Concept of Technology and Organizations », Organization Science, 3(3), 1992.

Orlikowski W, «Improvising Organizational Transformation Over Time: A Situated Change Perspective », Information Systems Research, 7(1), 1996, p. 63-92.

Orlikowski W, «Using Technology and Constituting Structures; A Practical lens for Studying Technology in Organizations ", Organization Science, 11(4), 2000, p. 404-428.à

Orlikowski W, «Material Works: Exploring the Situated Entanglement of Technological Performativity and Human Agency », Scandinavian Journal of Information Systems, 17(1), 2005, p. 183186. 
Orlikowski W, Robey, D, «Information Technology and the Structuring of Organizations », Information Systems Research, 2(2), 1991, p. 143-169.

Pène $\mathbf{S}$, Société de disponibilité. La vie quotidienne des communautés artificielles, HDR, Tome 2, Université Paris Descartes, 2005, 283p.

Poole M S, McPhee R D, «A Structurational Analysis of Organizational Climate », In L. L. Putnam \& M. E. Pacanowsky (Eds.), Communication and Organizations: An Interpretive approach, Newbury, CA: Sage Publications, 1983, p. 195-219.

Prenkert F, «A theory of organizing informed by activity theory: The locus of paradox, sources of change, and challenge to management », Journal of Organizational Change, 19(4), 2006, p. 471-490.

Putnam L, "Paradigms for Organizational Communication: An Overview and Synthesis ", The Western Journal of Speech Communication, 46 (printemps), 1982, p. 192-206.

Putnam L, Pacanowsky M E, Communication and Organizations; An Interpretive Approach, Newbury Park, Ca: Sage Publications, 1983.

Rojot J, (dir.), Revue de Gestion des Ressources Humaines, 1998, p. 26-27.

Roux A, Evolution des systèmes d'information et transformation des organisations: des dynamiques renouvelées, thèse de doctorat, Université Toulouse 2-Toulouse 3, 2003.

Rose J, Jones M, Truex D, «Socio-Theoretic Accounts of IS: The Problem of Agency », Scandinavian Journal of Information Systems, 17(1), 2005, p. 133-152.

Simondon G, L'invention dans les techniques. Cours et conférences, Seuil, 2005.

Suchman L (dir.) Computer-Supported Cooperative Work Conference Proceedings, Portland, Oregon, 1988.

Suchman L, Plans and Situated Action: The Problem of Humanmachine Communication, Cambridge MA: Cambridge University Press, 1987.

Walshman G, "Cross-Cultural Software Production and Use: A Structurational Analysis », MIS Quarterly, 26(4), 2002. 
Dossier : Migrations conceptuelles. D'où viennent les concepts de la communication organisationnelle?

Walshman G, "Agency Theory: Integration of a Thousand Flowers? », Scandinavian Journal of Information Systems, 17(1), 2005, p. 153-158.

Weick K E, «Organizational Communication: Toward a Research Agenda », in Putnam L, Pacanowsky M E (dir.), Communication and Organizations: An Interpretive Approach, Newbury Park, CA: Sage Publications, 1983, p. 13-29.

Weick K E, The Social Psychology of Organizing. New York, N.Y.: Random House, 1979.

Wilhelm C, «Diversité et co-construction interactive en ligne. L'étude de cas d'un dispositif d'apprentissage collaboratif à distance ", Colloque Pratiques et usages organisationnels des sciences et technologies de l'information et de la communication, Rennes, 2006, p. 252-258. 
